\title{
Morfologia polínica de espécies de Euphorbiaceae s.l. arbóreas ocorrentes no Estado de Santa Catarina, Brasil
}

\author{
Érika de Oliveira Sales ${ }^{1,4}$, Cintia Ferreira Barreto ${ }^{1,2}$ e Ortrud Monika Barth ${ }^{1,2,3}$
}

Recebido: 15.01.2009; aceito: 27.10.2011

\begin{abstract}
Pollen morphology of arboreal species of Euphorbiaceae s.l. at Santa Catarina State, Brazil). The pollen morphology of seven species of Euphorbiaceae occurring in the State of Santa Catarina, were examined: Actinostemon concolor, Hieronima alchorneoides, Maprounea brasiliensis, Pachystroma longifolium, Pausandra morisiana, Sapium glandulosum and Sebastiania argutidens. Except for Pausandra morisiana, all species have pollen grains in monads, isopolar, small to medium, radial symmetry, 3-colporate, oblate-spheroidal to prolate, and with a microreticulate ornamentation. Pollen grains of Pausandra morisiana follow the Croton-pattern. The morphometric data showed that Hieronima alchorneiodes pollen grains present the equatorial axis shorter than the other specimens studied. Pachystroma longifolium is the only species that shows a nexine thicker than the sexine, which characterizes pollen grains more resistant to the fossilization processes. Key words: Euphorbiaceae, Palynotaxonomy, pollen grains
\end{abstract}

RESUMO - (Morfologia polínica de espécies de Euphorbiaceae s.l. arbóreas ocorrentes no Estado de Santa Catarina, Brasil). Foram examinadas sete espécies de Euphorbiaceae s.l. que ocorrem no Estado de Santa Catarina, Brasil: Actinostemon concolor, Hieronima alchorneoides, Maprounea brasiliensis, Pachystroma longifolium, Pausandra morisiana, Sapium glandulosum e Sebastiania argutidens. Foram caracterizadas, exceto Pausandra morisiana, por apresentarem grãos de pólen em mônades, isopolares, pequenos a médios, de simetria radial, 3-colporados, oblato-esferoidais a prolatos e de superfície microrreticulada. Pausandra morisiana segue o padrão Croton. Os dados morfométricos mostraram que os grãos de pólen de Hieronima alchorneiodes apresentaram o eixo equatorial bem menor em relação às demais espécies estudadas. Pachystroma longifolium foi a única espécie que apresentou uma nexina mais espessa do que a sexina, o que caracteriza grãos de pólen como sendo mais resistentes a processos de fossilização.

Palavras-chave: Euporbiaceae, grãos de pólen, Palinotaxonomia

\section{Introdução}

Prosseguindo no estudo da morfologia polínica das espécies arbóreas de Euphorbiaceae s.l. ocorrentes no Brasil Meridional, em particular no Estado de Santa Catarina (Coelho et al. 2006), preferiu-se utilizar a mesma delimitação da família que havia sido utilizada no referido trabalho, embora que na literatura mais recente Phyllantaceae seja considerada um família a parte. Foram consideradas no presente trabalho sete espécies: Actinostemon concolor (Spreng.) Müll.Arg., Hieronima alchorneoides Allem. (Phyllanthaceae), Maprounea brasiliensis A.St.-Hil., Pachystroma longifolium (Nees) I.M.Johnst.,
Pausandra morisiana (Casar.) Radlk., Sapium glandulosum (L.) Morong e Sebastiania argutidens (Pax. \& K.Hoffm.). Segundo Smith et al. (1988), quatro são exclusivas da floresta ombrófila da encosta atlântica. Hieronima alchorneoides é encontrada com frequência em florestas secundárias, ocorrendo desde o Amazonas até o nordeste do Rio Grande do Sul; Pachystroma longifolium é heliófita, tem preferência a se desenvolver em encostas íngrimes e com floresta primária; Pausandra morisiana foi encontrada no interior de florestas primárias com terrenos úmidos e de drenagem lenta, sendo esciófita; Sebastiania argutidens está presente principalmente no interior de florestas aluviais, sendo rara na orla de matas e

1. Universidade Federal do Rio de Janeiro, Departamentos de Botânica e de Geologia, Laboratório de Palinologia, Cidade Universitária, Ilha do Fundão, 21949-900 Rio de Janeiro, Brasil

2. Universidade Federal do Rio de Janeiro, Instituto de Geociências, Curso de Pós-Graduação em Paleontologia e Estratigrafia

3. Instituto Oswaldo Cruz, Fiocruz, Avenida Brasil 4365, 21040-900 Rio de Janeiro, Brasil

4. Autor para correspondência: erika_melisso@yahoo.com.br 
em capoeiras. Duas espécies são consideradas mais expressivas na floresta estacional do alto rio Uruguai: Sapium glandulosum que ocorre também nas florestas dos pinhais, sendo heliófita, e Actinostemon concolor que é esciófita e pouco frequente nos bosques dos pinhais. Maprounea brasiliensis é restrita ao extremo nordeste de Santa Catarina. Porém, Lorenzi (1998) descreve-a como ocorrente em diversas formações vegetais, desde a floresta pluvial Amazônica até a Mata Atlântica no Estado do Paraná, no Cerrado e na mata semidecídua.

Apesar de haver vários estudos palinológicos descrevendo as características morfológicas de espécies pertencentes à família Euphorbiaceae, poucos foram os realizados com as espécies selecionadas para este trabalho. Cruz-Barros (2006), descreveu as características morfológicas das espécies Actinostemon concolor, Pausandra morisiana e Sebastiania corniculata da Ilha do Cardoso, São Paulo, Brasil. Salgado-Labouriau (1973) apresentou a morfologia do pólen de algumas espécies de Sapium, de Sebastiania e de Maprounea brasiliensis, enquanto que Smith et al. (1988) estudaram a taxonomia de diversas espécies de Euphorbiaceae do Estado de Santa Catarina.

O presente trabalho tem como objetivo descrever a morfologia de grãos de pólen de sete espécies de Euphorbiaceae ocorrentes no Estado de Santa Catarina, contribuindo assim para futuros trabalhos taxonômicos.

\section{Material e métodos}

O material polínico em estudo foi obtido do Herbário Barbosa Rodrigues, Itajaí, Santa Catarina, Brasil e do Herbário do Jardim Botânico do Rio de Janeiro, Brasil.

BRASIL. Santa Catarina: Brusque: Actinostemon concolor - Mata do Hoffmann, 3-X-1949, H.P. Veloso 55 (HBR), det. L.B. Smith \& Downs; Hieronima alchorneoides - Mata do Hoffmann,15-XII-1949, H.P. Veloso 35 (HBR), det. L.B. Smith \& Downs; Pachystroma longifolium Turvo Jacinto Machado, 6-XI-1951, H.P. Veloso 210 (HBR), det. L.B. Smith \& Downs; Pausandra morisiana - Mata do Hoffmann, 10-XI-1951. H.P. Veloso 64 (HBR), det. L.B. Smith \& Downs; Sapium glandulosum - Mata do Hofmann, 3-XI1949, H.P. Veloso 210 (HBR), det. L.B. Smith \& Downs; Sebastiania argutidens - Mata do Maluche,
11-XII-1951, H.P. Veloso 105 (HBR), det. L.B. Smith \& Downs. Rio DE JANEIRO: Maprounea brasiliensis, 18--VII-1950, A.P. Duarte 2808 (RB71701).

As anteras do material de herbário foram maceradas através de uma tela de metal e submetidas à acetólise clássica (Erdtman 1952). O pólen obtido foi incluído em gelatina glicerinada, lutando-se com parafina. As mensurações polínicas foram realizadas dentro do tempo máximo de sete dias após a acetólise (Salgado-Labouriau 1973). Foi usado tambor micrométrico Carl Zeiss, sendo medidos 25 grãos de pólen quanto aos eixos polar e equatorial e 10 grãos de pólen quanto à exina, ao colpo e retículo, todos escolhidos aleatoriamente. A partir destes resultados foram considerados grãos de pólen pequenos (10 a $25 \mu \mathrm{m}$ ), grãos de pólen médios (25 a 50 $\mu \mathrm{m})$, e grãos de pólen grandes (50 a $100 \mu \mathrm{m})$. As fotomicrografias foram obtidas com câmera analógica Sony acoplada a um microscópio de luz fotônica (ML) Axiophot, Zeiss e processadas no programa AxioVision 3.0 Zeiss. Foi utilizada a metodologia e terminologia proposta em Salgado-Labouriau (1973), Barth \& Melhem (1988) e Punt et al. (2007).

\section{Resultados e Discussão}

As descrições das características morfológicas das espécies examinadas de Euphorbiaceae seguem abaixo, enquanto que os dados morfométricos estão apresentados nas tabelas 1 e 2 .

Actinostemon concolor (Spreng.) Müll.Arg. Figuras 1-4

Grãos de pólen mônades, isopolares, pequenos, simetria radial, oblato-esferoidais, âmbito subcircular, 3-colporados, colpos longos e invaginados, endoaberturas lalongadas com margens mal definidas e exina microrreticulada.

Hieronima alchorneoides Allem. (Phyllanthaceae) Figuras 5-7

Grãos de pólen mônades, isopolares, pequenos, simetria radial, prolatos, âmbito subtriangular, 3-colporados, colpos longos, endoaberturas lalongadas e exina microrreticulada.

Maprounea brasiliensis A.St.-Hil. Figuras 8-11

Grãos de pólen mônades, isopolares, médios, simetria radial, prolatos, âmbito subtriangular, 
Tabela 1. Dados morfométricos dos grãos de pólen $(\mu \mathrm{m})$. n: 25, P: eixo polar, E: eixo equatorial e P/E: razão que indica a forma do grão de pólen.

Table 1. Morphometric data of pollen grains $(\mu \mathrm{m}) \mathrm{n}: 25$, P: polar axis, E: equatorial axis e P/E: ratio indicative of pollen grain form.

\begin{tabular}{lcccl}
\hline Espécies & $\mathrm{P}(\mu \mathrm{m})$ & $\mathrm{E}(\mu \mathrm{m})$ & $\mathrm{P} / \mathrm{E}$ & Forma \\
\hline Actinostemon concolor & $23,3 \pm 0,2$ & $23,8 \pm 0,3$ & 0,98 & oblato esferoidal \\
& $(21,0-25,2)$ & $(20,0-25,8)$ & & \\
Hieronima alchorneoides & $23,6 \pm 0,3$ & $12,3 \pm 0,2$ & 1,92 & prolato \\
& $(22,0-26,0)$ & $(10,8-13,6)$ & & \\
Maprounea brasiliensis & $25,4 \pm 0,3$ & $21,9 \pm 0,3$ & 1,16 & subprolato \\
& $(23,1-27,0)$ & $(15,4-25,8)$ & & \\
Pachystroma longifolium & $25,8 \pm 0,3$ & $23,7 \pm 0,3$ & 1,09 & prolato-esferoidal \\
Sapium glandulosum & $(23,4-27,3)$ & $(21,4-25,0)$ & & \\
Sebastiania argutidens & $24,1 \pm 0,2$ & $17,2 \pm 0,1$ & 1,33 & prolato \\
& $(22,0-26,8)$ & $(16,9-18,2)$ & & \\
Pausandra morisiana & $19,1 \pm 0,1$ & $17,6 \pm 0,1$ & 1,08 & prolato esferoidal \\
\hline
\end{tabular}

Tabela 2. Dados morfométricos dos grãos de pólen $(\mu \mathrm{m})$. S: espessura da sexina, N: espessura da nexina, C: comprimento do colpo e R: maior diâmetro do lúmen de retículo.

Table 2. Morphometric data of pollen grains $(\mu \mathrm{m})$. S: thickness of sexine, N: thickness of nexine, C: length of colpus and $\mathrm{R}$ : major diameter of lumina of reticulum.

\begin{tabular}{lcccc}
\hline Espécies & $\mathrm{S}(\mu \mathrm{m})$ & $\mathrm{N}(\mu \mathrm{m})$ & $\mathrm{C}(\mu \mathrm{m})$ & $\mathrm{R}(\mu \mathrm{m})$ \\
\hline Actinostemon concolor & 1,2 & 0,9 & 22,5 & 0,5 \\
Hieronima alchorneoides & 1,3 & 0,9 & 21,9 & \\
Maprounea brasiliensis & 1,5 & 0,7 & 20,9 & 0,4 \\
Pachystroma longifolium & 1,0 & 2,1 & 21,6 & 0,6 \\
Sapium glandulosum & 1,0 & 0,7 & 22,0 & 0,4 \\
Sebastiania argutidens & 2,1 & 1,2 & 14,9 & 0,5 \\
Pausandra morisiana & 8,6 & 1,0 & & \\
\hline
\end{tabular}

3-colporados, colpos longos e invaginados e endoaberturas ligeiramente lalongadas e exina microrreticulada.

\section{Pachystroma longifolium (Nees) I.M. Johnst.}

Figuras 12-17

Grãos de pólen mônades, isopolares, pequenos, simetria radial, subprolatos, âmbito subtriangular, 3-colporados, colpos longos, endoaberturas ligeiramente lolongadas com costas e exina microrreticulada.

Pausandra moresiana (Casar.) Radlk.

\section{Figuras 18-19}

Grãos de pólen mônades, apolares, grandes, simetria radial, esferoidais, âmbito circular, 
inaperturados, exina clavada em padrão-Croton, com 5-6 subunidades de ornamentação, sexina bastante espessa e nexina bem delgada.

Sapium glandulosum (L.) Morong [S. glandulatum (Vell.) Pax]

Figuras 20-22

Grãos de pólen mônades, isopolares, médios, simetria radial, prolatos, âmbito subcircular, 3-colporados, colpos longos, endoaberturas lolongadas com contorno pouco evidente, exina microrreticulada.

\section{Sebastiania argutidens Pax \& K.Hoffm.}

Figuras 23-27

Grãos de pólen mônades, isopolares, pequenos, simetria radial, oblato-esferoidais, âmbito subtriangular, 3-colporados, colpos longos
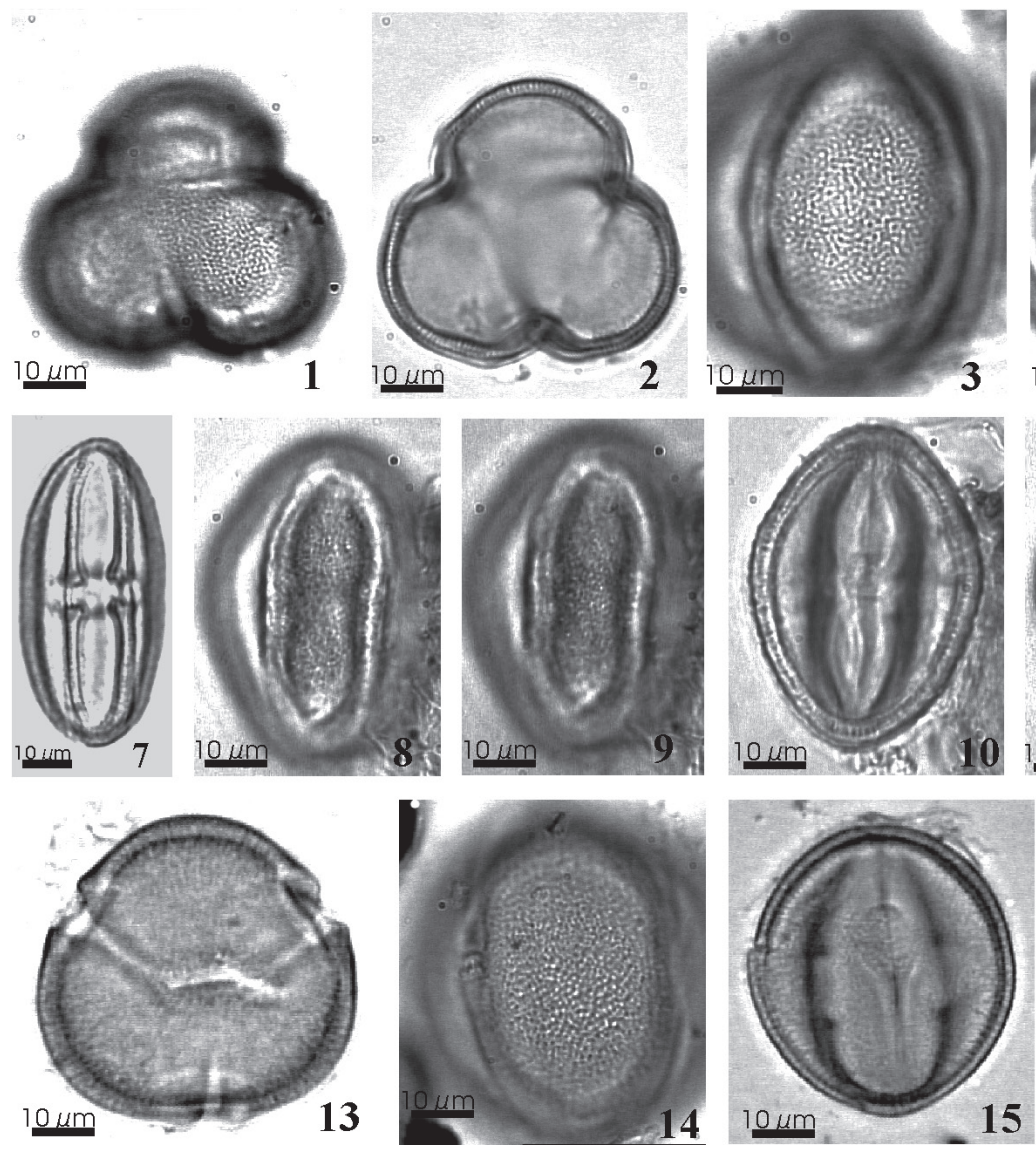
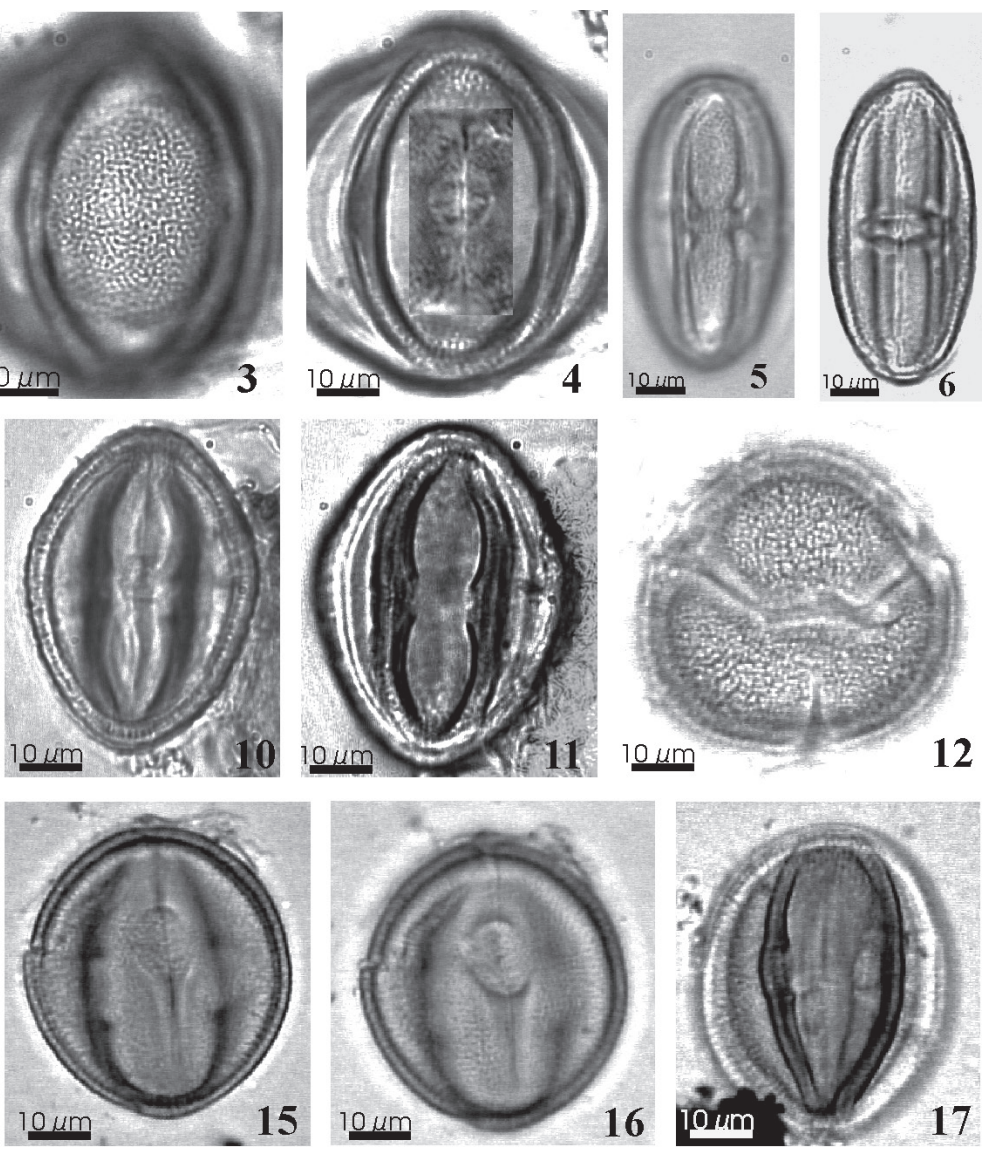

Figuras 1-17. Grãos de pólen de espécies de Euphorbiacaeae s.l. 1-4. Actinostemon concolor. 1. Vista polar, superficie. 2. Vista polar, corte óptico. 3. Vista equatorial, superfície. 4. Vista equatorial, dois colpos em corte longitudinal e cólporo. 5-7. Hieronima alchorneoides, vista equatorial. 5. Superficie. 6. Cólporo. 7. Cólporos em corte longitudinal. 8-11. Maprounea brasiliensis, vista equatorial. 8. Superfície em foco alto. 9. Superfície em foco baixo. 10. Corte óptico. 11. Cólporos em corte longitudinal. 12-17. Pachystroma longifolium. Grão de pólen rompido entre os cólporos. 12. Vista polar, evidenciando a superfície. 13. Vista polar, corte óptico. 14. Vista equatorial, superfície. 15. Vista equatorial, em corte óptico e evidenciando a endoabertura com costa. 16. Vista equatorial, cólporo. 17. Vista equatorial e cólporos em corte longitudinal.

Figures 1-17. Pollen grains of species of Euphorbiacaeae s.l. 1-4. Actinostemon concolor. 1. Polar view, surface. 2. Polar view, optical section. 3. Equatorial view, surface. 4. Equatorial view, longitudinal section of two colpi and colporus. 5-7. Hieronima alchorneoides, equatorial view. 5. Surface. 6. Colporus. 7. Colpori, longitudinal section. 8-11. Maprounea brasiliensis, equatorial view. 8. Surface, high focus. 9. Surface, low focus. 10. Optical section. 11. Colpori, longitudinal section. 12-17. Pachystroma longifolium. Pollen grain broken between the colpori. 12. polar view, surface. 13. polar view, optical section. 14. Equatorial view, surface. 15. Equatorial view, optical section and an endoaperture with costa. 16. Equatorial view, colporus. 17. Equatorial view, longitudinal section of colpori. 


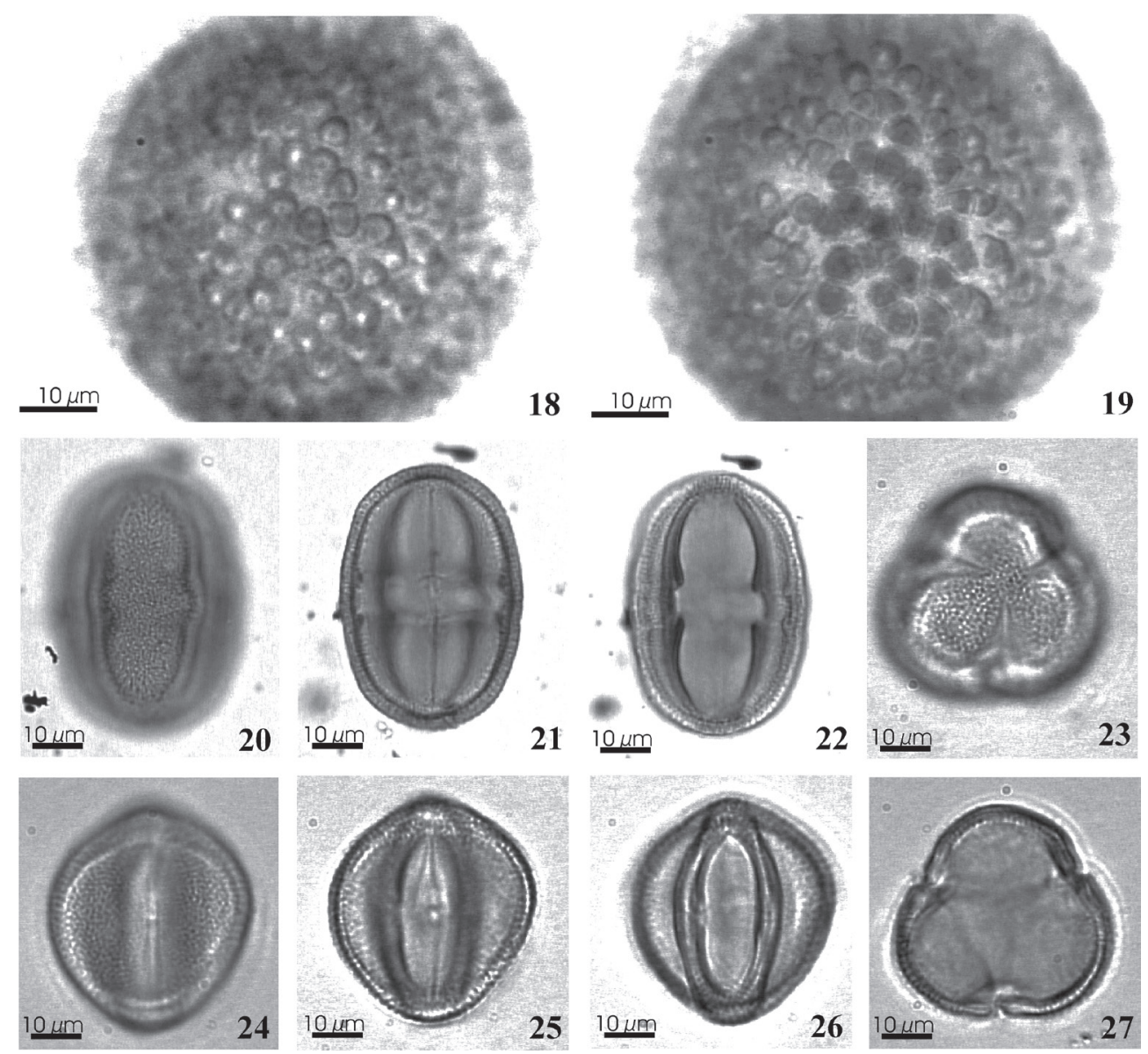

Figures 18-27. Grãos de pólen de espécies de Euphorbiacaeae s.l. 18-19. Pausandra morisiana. 18. Superfície em foco alto. 19. Superfície em foco baixo. 20-22. Sapium glandulosum, vista equatorial. 20. Superfície. 21. Corte óptico mostrando os cólporos. 22. Colpos em corte longitudinal. 23-27. Sebastiania argutidens. 23. Vista polar, superfície. 24. Vista equatorial evidenciando o cólporo. 25. Vista equatorial, corte óptico. 26. Vista equatorial, cólporos em corte longitudinal. 27. Vista polar, corte óptico, aberturas com fastígio.

Figuras 18-27. Pollen grains of species of Euphorbiacaeae s.l. 18-19. Pausandra morisiana. 18. Surface, high focus. 19. Surface, low focus. 20-22. Sapium glandulosum, equatorial view. 20. Surface. 21. Optical section showing the colpori. 22. Colpori, longitudinal section. 23-27. Sebastiania argutidens. 23. Polar view, surface. 24. Equatorial view showing the colporus. 25. Equatorial view, optical section. 26. Equatorial view, colpori, longitudinal section. 27. Polar view, optical section, apertures with fastigium.

Chave polínica para identificação das espécies de Euphorbiaceae

1. Grãos de pólen inaperturados

Pausandra morisiana

1. Grãos de pólen tricolporados

2. Oblato-esferoidais

3. Com presença de fastígio

Sebastiania argutidens

3. Sem presença de fastígio Actinostemon concolor

2. Subprolatos a prolatos

4. Subprolatos

Pachystroma longifolium

4. Prolatos

5. Relação P/E 1,92

Hieronima alchorneoides

5. Relação $\mathrm{P} / \mathrm{E}$ menor que 1,92

6. Eixo polar de 21,0 - 25,8 $\mu \mathrm{m}$

Sapium glandulosum

6. Eixo polar de 27,1 - 36,7 $\mu \mathrm{m}$ Maprounea brasiliensis 
e invaginados, endoaberturas com margens mal definidas, presença de fastígio, exina microrreticulada.

A morfologia dos grãos de pólen de Actinostemon concolor descrita no presente trabalho coincide com a descrição em Punt (1962), sendo pequenos e de forma oblata-esferoidal. Entretanto, Cruz-Barros et al. (2006) descreve-os como grãos médios e prolatoesferoidais. A superfície microrreticulada, assim como citada por Cruz-Barros et al. (2006), é diferente da citada em Punt (1962) que os descreve como psilados.

Pausandra morisiana apresentou grãos de pólen de tamanhos variáveis, tendo em média $75 \mu \mathrm{m}$ segundo Punt (1962), 49,3 $\mu \mathrm{m}$ segundo Cruz-Barros etal. (2006) e 59,6 $\mu \mathrm{m}$ no atual trabalho. A ornamentação da exina clavada, com padrão Croton de 5-6 subunidades, corrobora os resultados desses últimos autores. A nexina apresentou valores parecidos com os em Cruz-Barros et al. (2006), com a sexina bem mais espessa, caracterizando os grãos de pólen como frágeis por sua nexina relativamente fina.

A morfologia polínica de Maprounea brasiliensis apresentou características semelhantes às de Punt (1962) e Salgado-Labouriau (1973) que a descrevem como sendo subprolato a prolato, endoaberturas lolongadas, superfície microrreticulada e sexina com o dobro da espessura da nexina.

Punt (1962) estudou o pólen de Pachystroma longifolium (Nees). As medidas dos eixos polar e equatorial e sua razão apresentaram valores parecidos como os do presente estudo, entretanto apresentaram variações do comprimento dos colpos e a superfície da exina sendo psilada, enquanto no presente estudo esta é microrreticulada.

As características morfológicas dos grãos de Hieronima alchorneoides conferem com os descritos em Punt (1962) exceto nas medidas do eixo polar que se apresentou maior do que nos do presente trabalho.

\section{Agradecimentos}

Ao Conselho Nacional de Desenvolvimento Científico e Tecnológico (CNPq) pela bolsa de pesquisa concedida à última autora (Processo: 305752/2006-5).

\section{Literatura citada}

Barth, O.M. \& Melhem, T.S. 1988. Glossário ilustrado de Palinologia. Unicamp, Campinas.

Coelho, L.G. \& Barth, O.M. 2002. Catálogo sistempatico de pólen das plantas arbóreas do Brasil Meridional. XXXV - Euphorbiaceae: Alchornea, Aparisthmium, Bernardia e Pera. Leandra, 17: 35-44.

Cruz-Barros, M.A.V., Corrêa, A.M.S. \& MakinoWatanabe, H. 2006. Estudo polínico das espécies de Aquifoliaceae, Euphorbiaceae, Lecythidaceae, Malvaceae, Phytolaccaceae e Portulacaceae ocorrentes na restinga da Ilha do Cardoso. Revista Brasileira de Botânica 29: 145-162.

Erdtman, G. 1952. Pollen morphology and plant taxonomy-Angiosperms. Almqvist \& Wiksell, Stockholm.

Lorenzi, H. 1998. Árvores brasileiras. v. 2. Editora Plantarum, Nova Odessa.

Punt, W. 1962. Pollen morphology of the Euphorbiaceae with special reference to taxonomy. Botanical Museum and Herbarium, Utrecht.

Punt, W., Hoen, P.P., Blackmore, S., Nilsson, S. \& Le Thomas, A. 2007. Glossary of pollen and spore terminology. Review of Palaeobotany and Palynology 143: 1-8.

Salgado-Labouriau, M.L. 1973. Contribuição à palinologia dos Cerrados. Academia Brasileira de Ciências, Rio de Janeiro.

Smith, L.B., Downs, R.J. \& Klein, R.M. 1988. Euforbiáceas. In: R. Reitz (ed.). Flora Ilustrada Catarinense, Itajaí, pp. 1-408. 